\title{
GIANI: open-source software for automated analysis of 3D microscopy images
}

David J. Barry ${ }^{1 *}$, Claudia Gerri ${ }^{2}$, Donald M. Bell ${ }^{1}$, Rocco D’Antuono ${ }^{1}$, Kathy K. Niakan $^{2}$

1 Crick Advanced Light Microscopy, Francis Crick Institute, London, NW1 1ST, UK

2 Human Embryo and Stem Cell Laboratory, Francis Crick Institute, London, NW1 1ST, UK

*david.barry@crick.ac.uk 


\section{Abstract}

The study of cellular and developmental processes in physiologically relevant three-dimensional (3D) systems facilitates an understanding of mechanisms underlying cell fate, disease and injury. While cutting-edge microscopy technologies permit the routine acquisition of 3D datasets, there is currently a lack of user-friendly, open-source software to analyse such images. Here we describe GIANI (djpbarry.github.io/Giani), new software for the analysis of 3D images, implemented as a plugin for the popular FIJI platform. The design primarily facilitates segmentation of nuclei and cells, followed by quantification of morphology and protein expression. GIANI enables routine and reproducible batch-processing of large numbers of images and also comes with scripting and command line tools, such that users can incorporate its functionality into their own scripts and easily run GIANI on a high-performance computing cluster. We demonstrate the utility of GIANI by quantifying cell morphology and protein expression in mouse early embryos. More generally, we anticipate that GIANI will be a useful tool for researchers in a variety of biomedical fields.

\section{Introduction}

The ability to routinely acquire multi-dimensional datasets with modern microscopy techniques is transforming, among other fields, cell biology, developmental biology and cancer research. There has long been an acceptance that two-dimensional (2D) cell cultures may not accurately recreate behaviours found in complex, three-dimensional (3D) in vivo environments [1].

Commonly-used 3D culture formats include, but are not limited to, populations of single cells in organotypic matrices, spheroid models, tissue sections or 
whole embryos and organisms.

9

However, the development of user-friendly software for the quantitative $\quad 10$ analysis of such data has not kept pace with imaging advances [2]. This presents 11 an obvious challenge - how does one quantitatively analyse these datasets in a $\quad{ }_{12}$ routine manner? Manual annotation of such data is not feasible, due to the time ${ }_{13}$ required to do so. Commercial packages, such as Imaris (Bitplane) and Vison4D ${ }_{14}$ (Arivis) provide excellent visualisation functionality and are also equipped with $\quad 15$ analysis tools. However, licenses for such software are expensive and they also 16 rely on their own proprietary file formats. Furthermore, the closed-source nature $\quad 17$ of such software prevents detailed interrogation of specific calculations and $\quad 18$ processes.

There are numerous excellent, freely-available bioimage analysis tools in the $\quad 20$ open source domain. However, their support for 3D analysis is either limited $\quad{ }_{21}$ (for example, several CellProfiler [3] modules are not yet compatible with 3D 22 images) or challenging to execute and/or automate for the uninitiated (FIJI [4], 23 Icy [5]). This can lead to undesirable compromises being made, such as 2D $\quad 24$ slices from a 3D volume being analysed individually, blinding the analysis to $\quad 25$ information in adjacent slices. Alternatively, 3D data may be compressed into $\quad 26$ $2 \mathrm{D}$ via projection, which, consequently, artificially reduces distances between $\quad 27$ objects. A number of very useful open-source MATLAB (MathWorks, 28 Cambridge, UK) -based tools have also been implemented, most notably MINS, $\quad 29$ which has been effectively used to analyse embryo datasets [6] and 30 LOBSTER [7]. However, these require the purchase of a MATLAB license and 31 active maintenance of the code by the developers to avoid problems with 32 backwards compatibility in newer versions of MATLAB. 33

We have therefore developed GIANI (https://djpbarry.github.io/Giani), a 34 
user-friendly, generally-applicable, open source tool, implemented as a plugin $\quad 35$ for the widely-used image analysis platform, FIJI. With an emphasis on $\quad 36$ detection and segmentation of cells in 3D microscopy images, GIANI has been $\quad 37$ implemented specifically with batch-processing and minimal user interaction in $\quad 38$ mind. While an understanding of fundamental concepts of bioimage analysis is 39 beneficial, GIANI has been implemented in a wizard format to facilitate use by 40 non-specialists and is fully documented (https://github.com/djpbarry/Giani/wiki). ${ }_{41}$ Analysis protocols may be reproduced by loading a single, small parameter file. $\quad 42$

The utility of GIANI is illustrated here using two examples. In the first, we 43 generated a series of simulated datasets to evaluate the accuracy of $\quad 44$ segmentations produced by GIANI using known "ground truths". In the second 45 proof-of-concept we use a series of mouse preimplantation embryo datasets and $\quad 46$ demonstrate the ability of GIANI to detect variations in morphology and protein $\quad 47$ expression in different experimental conditions. It should be noted that GIANI $\quad 48$ can be used to quantify 3D images from a range of cellular and developmental $\quad 49$ contexts and we anticipate that it will be a useful tool to automate quantification $\quad 50$ of a wide variety of complex imaging data.

\section{Materials and methods}

\section{Simulated data generation}

Simulated embryos were generated using an extension of a previously described method [8]. Simulated nuclei positions were generated as previously 54 described [8], then cell membranes were approximated using a Euclidean Distance Map constructed around nuclei. The simulated images were then convolved with a Gaussian point-spread function, sub-sampled and noise added 
from a gamma distribution. The complete code for simulated image generation $\quad 59$ is available on GitHub (https://github.com/djpbarry/Embryo-Generator).

\section{Metrics for segmentation quality assessment}

We calculated the cell count error $E_{c}$, as

$$
E_{c}=\left|N_{g}-N_{g t}\right|
$$

where $N_{g t}$ is the actual number of cells present in a simulated embryo and $N_{g}$ is $\quad{ }_{63}$ the number counted by GIANI. Nuclear $\left(E_{n l}\right)$ and cell $\left(E_{c l}\right)$ centroid localisation $\quad 64$ errors were calculated based on the euclidean distance between the known $\quad 65$ ground truth centroids and the centroids of the segmentations produced by 66 GIANI.

\section{Mouse zygote collection}

Four- to eight-week-old (C57BL6 $\times$ CBA) F1 female mice were super-ovulated $\quad 69$ using injection of $5 \mathrm{IU}$ of pregnant mare serum gonadotrophin (PMSG; $\quad 70$ Sigma-Aldrich). Forty-eight hours after PMSG injection, 5 IU of human 71 chorionic gonadotrophin (HCG; Sigma-Aldrich) was administered. Superovulated females were set up for mating with eight-week-old or older $(\mathrm{C} 57 \mathrm{BL} 6 \times \mathrm{CBA}) \mathrm{F} 1$ males. Mice were maintained on a $12 \mathrm{~h}$ light-dark cycle. $\quad 74$ Mouse zygotes were isolated in EmbryoMax FHM mouse embryo media (Sigma-Aldrich; MR-122-D) under mineral oil (Origio; ART-4008-5P) and cumulus cells were removed with hyaluronidase (Sigma-Aldrich; H4272). All 77 animal research was performed in compliance with the UK Home Office 
Licence Number 70/8560.

\section{Mouse embryo culture}

Mouse embryos were cultured in drops of pre-equilibrated Global medium

(LifeGlobal; LGGG-20) supplemented with $5 \mathrm{mg} / \mathrm{ml}$ protein supplement

(LifeGlobal; LGPS-605) and overlaid with mineral oil (Origio; ART-4008-5P).

Preimplantation embryos were incubated at $37^{\circ} \mathrm{C}$ and $5.5 \% \mathrm{CO}_{2}$ and cultured up $\quad 84$ to the day of analysis.

\section{Inhibitor treatment}

Inhibitor experiment was performed as previously described [9]. Briefly, the 87 aPKC inhibitor CRT0276121 (Cancer Research Technology LTD) was dissolved in DMSO to $10 \mathrm{mM}$ stock concentration and diluted to the optimal concentration of $8 \mu \mathrm{M}$ in pre-equilibrated embryo culture media. Control mouse $\quad 90$ embryos were developed in pre-equilibrated media where the same amount of DMSO was added.

\section{Immunofluorescence}

Embryos were fixed with freshly prepared 4\% paraformaldehyde in PBS that was pre-chilled at $4^{\circ} \mathrm{C}$. Embryo fixation was performed for $20 \mathrm{~min}$ at RT and then the embryos were transferred through 3 washes of $1 \mathrm{X}$ PBS with $0.1 \%$ Tween-20 to remove residual paraformaldehyde. Embryos were permeabilized with $1 \mathrm{X}$ PBS with $0.5 \%$ Triton X-100 and then blocked in blocking solution (3\% BSA in $1 \mathrm{X}$ PBS with $0.2 \%$ Triton $\mathrm{X}-100$ ) for $2 \mathrm{~h}$ at $\mathrm{RT}$ on a rotating shaker. Then, embryos were incubated with primary antibodies (listed in Table 1) 
Table 1. Primary antibodies used in this study and the dilution at which they were used.

\begin{tabular}{llll}
\hline Antibody & Supplier & Catalogue Number & Dilution \\
\hline YAP1 & Abnova & H00010413-M01 & $1: 50$ \\
GATA3 & R\&D & AF2605 & $1: 200$ \\
E-CADHERIN & Life Technologies & 131900 & $1: 400$ \\
\hline
\end{tabular}

The following day, embryos were washed in 1X PBS with 0.2\% Triton X-100 102 for 20 min at RT on rotating shaker and then incubated with secondary antibodies diluted in blocking solution for $1 \mathrm{~h}$ at RT on rotating shaker in the dark. Next, embryos were washed in $1 \mathrm{X}$ PBS with $0.2 \%$ Triton X-100 for 20 min at RT on rotating shaker. Finally, embryos were placed in 1X PBS with 0.1\% Tween-20 with Vectashield with DAPI mounting medium (Vector Lab; 107 H-1200) (1:30 dilution). Embryos were placed on $\mu$-Slide 8 well dishes (Ibidi; $\quad 108$ 80826) for confocal imaging.

\section{Image Acquisition}

All images were acquired on a Leica SP5 laser scanning confocal microscope $\quad 111$ using a Leica 1.3 NA 63x HCX PL APO CS glycerol objective and a voxel size 112 of approximately $0.1 \times 0.1 \times 1.0 \mu \mathrm{m}$ in $\mathrm{x}, \mathrm{y}$ and $\mathrm{z}$, respectively.

\section{Software}

GIANI was written using Java 8 as a plug-in for FIJI [4], making extensive use ${ }_{115}$ of the underlying FIJI and ImageJ [10] libraries. A number of other open-source 116 projects are leveraged. Reading of image data is facilitated by interfacing to 117 Bio-Formats [11]. Detection of nuclear blobs makes use of either TrackMate's ${ }_{118}$

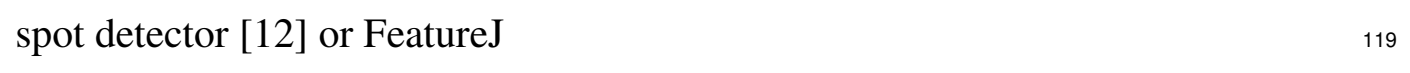
(https://imagescience.org/meijering/software/featurej). Segmentation of cells $\quad{ }_{120}$ 
and nuclei takes advantage of the marker-controlled watershed functionality in $\quad 121$ MorphoLibJ [13] and 3D Image Suite [14]. The browsing of results is based 122 upon the 3D Region of Interest (ROI) Manager from 3D Image Suite. Complete $\quad 123$ source code, documentation and test data are available online $\quad 124$ (https://djpbarry.github.io/Giani).

\section{Software implementation}

The design philosophy behind GIANI is inspired by CellProfiler. It is assumed ${ }_{128}$ that the user wishes to detect "primary objects" of some sort (typically cell ${ }_{129}$ nuclei), followed by the subsequent segmentation of "secondary objects" $\quad 130$ (typically cells) and then wishes to measure either the morphology of said 131 objects, or the expression of a fluorescent signal within these objects. The 132 principal difference in the case of GIANI is that, in order to facilitate ease of use, 133 the order of steps in the pipeline is fixed, although some flexibility is present $\quad{ }_{134}$ where necessary (Fig 1).

\section{Analysis of simulated embryos}

To first validate the performance of GIANI, we generated a series of 189

"simulated embryos" exhibiting different levels of signal-to-noise ratio and cell density (Fig 2A). Simulated data has the significant advantage of having a known "ground truth" (Fig 2B). That is, because the images are generated artificially, we know what the "correct" segmentation should look like. This 
Fig 1. Overview of GIANI's operation All embryo images represent a single slice of a 3D stack. A: The GIANI Graphical User Interface. B: An example dataset, consisting of a 3D stack image of a mouse embryo showing DAPI (blue), E-Cadherin (red) and GATA-3 (green). C: Positions of nuclei are first approximated using a blob detector, which provides an estimate of each nuclear centroid. D: Prior to complete segmentation, the nuclear image stack is Gaussian filtered in three dimensions. E: Complete segmentation of the nuclei is achieved using a marker-controlled watershed approach, with the filtered image from $D$ serving as the input and centroids from $C$ serving as the markers. The extent of the nuclei is based on an intensity threshold, calculated using one of FIJI's in-built thresholding algorithms, specified by the user. F: Prior to conducting cell segmentation, the image channel delineating cells is Gaussian filtered in three dimensions. Either a volume marker or membrane marker can be used, but the latter will result in more accurate segmentation. G: Cell segmentation is achieved using a marker-controlled watershed approach, with the filtered image from $\mathrm{F}$ serving as the input and nuclei segmentations from $\mathrm{E}$ serving as the markers. The extent of the cells is again based on an intensity threshold, calculated using one of FIJI's thresholding algorithms. H: Quantification of fluorescence intensities in any number of user-specified channels is performed using the segmentations from $\mathrm{E}$ and $\mathrm{G}$. Results are generated for each nucleus, cell and cytoplasmic region in each input stack. I: Once a batch analysis has been completed, segmentations and associated fluorescence quantifications can be explored using the provided results browser.

Fig 2. Validation of GIANI using simulated embryo data. In each of the heat maps, a single tile represents the average of three simulated embryos. A: A 2D slice of an exemplar 3D simulated embryo. B: The ground truth segmentation of 'A'. C - H: Absolute errors in cell counts $\left(E_{c}\right.$, calculated according to Eq 1), nuclear centroid localisation error $\left(E_{n l}\right)$ and cell centroid localisation error $\left(E_{c l}\right)$ produced by GIANI for simulated embryos with the indicated number of cells and signal-to-noise ratios (SNR). Results were obtained using either GIANI's basic $(\mathrm{C}-\mathrm{E})$ or advanced $(\mathrm{F}-\mathrm{H})$ nuclear detector.

The number of false positives and false negatives produced by GIANI is consistently extremely low, although cell count errors do generally increase with cell density and decreasing signal-to-noise ratio (SNR; Fig 2 C, F). At high SNR and low cell densities, GIANI localises nuclei to within approximately $600 \mathrm{~nm}$ of their true positions (Fig 2D, G). Even at high cell densities and low SNR, this error never exceeded $1 \mu \mathrm{m}$ (the simulated nuclei are ellipsoidal with axis 
dimensions of approximately $10.0 \times 7.5 \times 7.5 \mu \mathrm{m})$. In addition to nuclear $\quad 150$ localisation error, we also quantified the cell centroid localisation error on a per 151 cell basis (Fig 2E, H).

The accurate characterisation of a multi-cellular dataset, such as an embryo, 153 is fundamentally dependent on the correct identification and segmentation of $\quad 154$ nuclei. While GIANI presently employs generic blob detectors for this purpose, 155 which is similar to other previously-described methods [6], other approaches are ${ }_{156}$ also possible [15-19]. It is intended that some alternative functionality will be $\quad{ }_{157}$ added in future releases. In particular, incorporating machine-learning classifiers $\quad 158$ trained with, for example, Weka [20] or Ilastik [21] will be prioritised.

In addition to the convolution of the ground truth data, the simulation process 160 also takes into consideration depth of slices within a sample when modelling $\quad{ }_{161}$ fluorescent intensity - deeper slices will be more prone to scattering effects and 162 therefor exhibit lower intensity. This means that finding a single global thresholding strategy that does not under-segment the dimmer cells while also not over-segmenting the brighter cells is challenging. Taking all this into 164 consideration, any metric used to assess the accuracy of cell segmentations will have its limitations.

There are strategies that could be employed to mitigate against these factors. 168 For example, some form of adaptive thresholding, whereby the intensity optimal adaptive thresholding strategy could be found for a given dataset (such $\quad 171$ as the simulated data used in this study), implementing a universally-applicable 172 strategy would be difficult. One of the principle design aims of GIANI was 173 simplicity of use, which does not allow for the incorporation of a variety of $\quad 174$ case-specific segmentation approaches. 


\section{Analysis of mouse embryos}

We subsequently applied GIANI to the analysis of two populations of mouse

preimplantation embryos at the morula stage, one control and one treated with a 178 small molecule inhibitor [9]. At this stage, two distinct cell populations are $\quad 179$ discernible: inner and outer cells. In subsequent cell divisions, a blastocyst is $\quad 180$ formed, whereby the inner cells give rise to an inner cell mass (ICM), and the ${ }_{181}$ outer cells become the trophectoderm (TE), a polarized epithelium that will $\quad 182$ form fetal components of the placenta [22].

At the morula stage, inner and outer cells display different polarisation states, 184 which influence their cell fate acquisition. The outer cells acquire an apical 185 domain, enriched with the atypical protein kinase C (aPKC) [23]. In the polar ${ }_{186}$ outer cells, aPKC prevents activation of downstream Hippo pathway kinases, $\quad{ }_{187}$ Large tumor suppressor kinases 1/2 (LATS1/2) [24]. Consequently, in outer 188 cells, YAP1 accumulates in the nucleus, where it promotes the expression of 189 GATA3 [25]. In contrast, in the apolar inner cells, the activation of the Hippo 190 pathway results in YAP1 cytoplasmic retention, thus maintaining the inner cells 191 in an unspecified state [24,26, 27].

We therefore divided cells within each embryo into an 'inner' and 'outer' 193 population (Fig 3A, B). This division is based on the distance of the detected 194 nuclear centroid from the embryo centroid. A cell was classified as 'inner' if the 195 following condition held true:

$$
\frac{D_{i}}{D_{m}}<D_{T}
$$

where $D_{i}$ is the Euclidean distance of cell $i$ from the embryo centroid, $D_{m}$ is the 197 
maximum Euclidean distance from any cell in the same embryo to the embryo $\quad 198$ centroid and $D_{T}$ is an arbitrarily set distance threshold. For the purposes of this 199 study, we chose a value of 0.5 for $D_{T}$ - it was found that modifying this value $\quad 200$ slightly $( \pm 10 \%)$ did not significantly impact the results (data not shown). The $\quad 201$ embryo centroid was calculated as the average of all nuclear centroids.

Fig 3. GIANI reveals differences in morphology and protein expression in mouse embryos. All box plots show the median and inter-quartile range, with the whiskers extending 1.5 times the inter-quartile range from the 25 th and 75 th percentiles. Unless otherwise stated, each dot represents a single cell. A. Illustration of the division of embryo cells into 'outer' (red) and 'inner' (green) sub-populations. The embryo centroid is indicated by the white square. The blue circle has radius $D_{m}$ (see $\mathrm{Eq} 2$ ) and indicates the distance from the embryo centroid to the most distant nucleus centroid. The radius of the yellow circle is $D_{T} \times D_{m}(\mathrm{Eq} 2)$. B. The number of cells in each embryo divided into outer and inner sub-populations using a value of 0.5 for $D_{T}$ in control embryos. Each dot represents a single embryo $\left(n_{\text {control }}=18\right)$. C: Volume of nuclei in inner/outer populations in control embryos. D: Volume of cells in inner/outer populations in control embryos. E: Ratio of cell-to-nuclear volume in inner and outer cells in control embryos. F: Nuclear/cytoplasmic ratio of YAP1 expression in control embryos. G: Difference in expression profiles of nuclear GATA3 expression, normalised to DAPI, in control and treated embryos $\left(n_{\text {treated }}=20\right)$. H:

Difference in nuclear/cytoplasmic ratiometric expression profiles of YAP1 in control and treated embryos.

\section{GIANI shows differences in cell morphology, YAP1 and GATA3} expression within inner and outer cells in control embryos

We then applied GIANI to detect variations in morphology and protein

expression in mouse preimplantation embryos. We began with a morphological 206 analysis of cells in control embryos. While no significant difference was found between median nuclear volumes in inner and outer cell populations 207 (Fig $3 \mathrm{C} ; p=0.861$, Wilcoxon rank sum test), a difference in size distributions is evident. A difference in median cell volume between the two populations was also identified, although it fell slightly short of being statistically significant 
(Fig $3 \mathrm{D} ; p=0.069)$. However, a comparison of cell-to-nucleus volume ratios $\quad 212$ revealed a significant difference between median values, with outer cells having $\quad 213$ a proportionately greater cytoplasmic volume than inner cells (Fig $3 \mathrm{E} ; \quad 214$ $p<0.001)$. We also confirmed previous analysis of YAP1 translocation [28], $\quad 215$ illustrating that nuclear localisation is significantly higher in outer versus inner $\quad 216$ cells (Fig 3F; $p<0.001$ ). Similarly, GATA3 expression was shown to be higher 217 in outer compared to inner cells (Fig $3 \mathrm{G} ; p<0.0001$ ).

\section{GIANI reveals differences in YAP1 and GATA3 expression in mouse}

To further demonstrate the utility of GIANI, we sought to analyse GATA3 and $\quad{ }_{221}$ YAP1 expression after treating mouse embryos with a small molecular inhibitor 222 against aPKC, the upstream regulator of YAP1 and GATA3. The aPKC inhibitor, 223 CRT0276121, has previously been confirmed to specifically inhibit aPKC in $\quad 224$ various biological and cellular contexts [29-31]. Specifically, in mouse

efficiently abrogate YAP1 and GATA3 expression in outer cells [9].

Expression of GATA3 in the nucleus (normalised to DAPI to correct for diminished signal intensity with increasing sample depth) was found to be similarly low in the inner cells of both control and treated embryos (Fig $3 \mathrm{G}$; 
embryos, with two distinct populations evident in both inner and outer cells in $\quad 237$ control populations (Fig $3 \mathrm{G})$.

While no statistically significant difference in nuclear YAP1 expression $\quad 239$ (normalised to cytoplasmic expression) between control and treated cells were $\quad 240$ observed when comparing inner cells (Fig $3 \mathrm{H} ; p=0.609$ ), a large difference $\quad{ }_{241}$ was observed in outer cells $(p<0.0001)$. However, nuclear/cytoplasmic YAP1 ${ }_{242}$ expression was still higher in the outer cells of treated embryos versus inner $\quad{ }_{243}$ $(p<0.0001)$. Altogether, this demonstrates that GIANI allows for the 244 automated quantification of expression differences between cells following $\quad 245$ perturbation.

\section{Future developments}

Given the ongoing interest in studying cells in "native" 3D extracellular environments [32], future extension to the capabilities of GIANI will include the ${ }_{249}$ ability to analyse time-lapse data, as there is currently a lack of open source $\quad 250$ tools for the quantification of 3D cell migration [33]. The incorporation of $\quad 251$ additional functionality from TrackMate (and MaMuT [34] or Mastodon) will 252 be explored to facilitate 3D cell tracking.

Future development will also include the replacement of the 3D ROI Manager interface for visualizing results with a new, custom-built interface. $\quad 255$ This will probably be replaced in a subsequent release with a new custom-built $\quad 256$ interface. At present, segmented objects are saved solely as FIJI ROI files, but it ${ }_{257}$ is intended that support for other formats (such as .stl, .ply, .obj and .x3d) will 258 be added to allow the import of objects into a variety of different software. $\quad 259$

More generally, with a view to improving and optimising performance, $\quad 260$ further use of ImgLib2 [35] will be incorporated in future releases. Taking 261 
advantage of GPU acceleration is also an aim, most likely by exploiting

CLIJ [36]. For this study, analysis of a single mouse embryo dataset (approx. $\quad 263$

1024 x 1024 x 76 voxels) took approximately 5 minutes on a desktop PC (Intel 264

Xeon E5-2630 v4, 32GB RAM) versus 10 minutes on a laptop (Intel Core $\quad 265$

i7-6600U, 16 GB RAM).

\section{Conclusion}

We have used GIANI to quantitatively analyse mouse embryos in 3D. This

analysis has revealed differences in morphology and protein expression between 269 different experimental conditions. Analysis of simulated ground truth data was 270 used to confirm the validity of these results. Further development of GIANI is $\quad 271$ planned, with the specific aim of improving segmentations in noisy and dense $\quad 272$ fields of view, common in 3D images of cells. Extension to timelapse analysis is 273 also planned. GIANI is freely available on GitHub (github.com/djpbarry/Giani) ${ }^{274}$ and we anticipate that it will be a useful resource for the community to perform 275 routine, automated quantification of complex imaging data.

\section{Acknowledgments}

Thanks to Kurt Anderson for his constructive comments on the manuscript.

\section{References}

1. Duval K, Grover H, Han LH, Mou Y, Pegoraro AF, Fredberg J, et al. Modeling Physiological Events in 2D vs. 3D Cell Culture. Physiology. 2017;32(4):266-277. 
2. Meijering E, Carpenter AE, Peng H, Hamprecht FA, Olivo-Marin JC. Imagining the future of bioimage analysis. Nature Biotechnology. 2016;34(12):1250-1255.

3. Lamprecht MR, Sabatini DM, Carpenter AE. CellProfiler ${ }^{\mathrm{TM}}$ : free, versatile software for automated biological image analysis.

BioTechniques. 2007;42(1):71-75.

4. Schindelin J, Arganda-Carreras I, Frise E, Kaynig V, Longair M, Pietzsch T, et al. Fiji: an open-source platform for biological-image analysis. Nature Methods. 2012;9:676.

5. de Chaumont F, Dallongeville S, Chenouard N, Hervé N, Pop S, Provoost T, et al. Icy: an open bioimage informatics platform for extended reproducible research. Nature Methods. 2012;9:690.

6. Lou X, Kang M, Xenopoulos P, Muñoz-Descalzo S, Hadjantonakis AK. A Rapid and Efficient 2D/3D Nuclear Segmentation Method for Analysis of Early Mouse Embryo and Stem Cell Image Data. Stem Cell Reports. 2014;2(3):382 - 397.

7. Tosi S, Bardia L, Filgueira MJ, Calon A, Colombelli J. LOBSTER: An environment to design bioimage analysis workflows for large and complex fluorescence microscopy data. Bioinformatics. 2019;

8. Rajasekaran B, Uriu K, Valentin G, Tinevez JY, Oates AC. Object Segmentation and Ground Truth in 3D Embryonic Imaging. PLOS ONE. 2016;11(6):1-17. 
9. Gerri C, McCarthy A, Alanis-Lobato G, Demtschenko A, Bruneau A, Loubersac $\mathrm{S}$, et al. Initiation of a conserved trophectoderm program in human, cow and mouse embryos. Nature. 2020;.

10. Schneider CA, Rasband WS, Eliceiri KW. NIH Image to ImageJ: 25 years of image analysis. Nature methods. 2012;9:671-675.

11. Linkert M, Rueden CT, Allan C, Burel JM, Moore W, Patterson A, et al. Metadata matters: access to image data in the real world. The Journal of Cell Biology. 2010;189(5):777-782.

12. Tinevez JY, Perry N, Schindelin J, Hoopes GM, Reynolds GD, Laplantine E, et al. TrackMate: An open and extensible platform for single-particle tracking. Methods. 2017;115:80 - 90.

13. Legland D, Arganda-Carreras I, Andrey P. MorphoLibJ: integrated library and plugins for mathematical morphology with ImageJ. Bioinformatics. 2016;32(22):3532-3534.

14. Ollion J, Cochennec J, Loll F, Escudé C, Boudier T. TANGO: a generic tool for high-throughput 3D image analysis for studying nuclear organization. Bioinformatics. 2013;29:1840-1841.

15. Kostrykin L, Schnörr C, Rohr K. Globally optimal segmentation of cell nuclei in fluorescence microscopy images using shape and intensity information. Medical Image Analysis. 2019;58:101536.

16. Weigert M, Schmidt U, Haase R, Sugawara K, Myers G. Star-convex Polyhedra for 3D Object Detection and Segmentation in Microscopy; 2019. 
17. Blin G, Sadurska D, Portero Migueles R, Chen N, Watson JA, Lowell S. Nessys: A new set of tools for the automated detection of nuclei within intact tissues and dense 3D cultures. PLOS Biology. 2019;17(8):1-29.

18. Caicedo JC, Goodman A, Karhohs KW, Cimini BA, Ackerman J, Haghighi M, et al. Nucleus segmentation across imaging experiments: the 2018 Data Science Bowl. Nature Methods. 2019;.

19. Yang L, Ghosh RP, Franklin JM, Chen S, You C, Narayan RR, et al. NuSeT: A deep learning tool for reliably separating and analyzing crowded cells. PLOS Computational Biology. 2020;16(9):1-20.

20. Arganda-Carreras I, Kaynig V, Rueden C, Eliceiri KW, Schindelin J, Cardona A, et al. Trainable Weka Segmentation: a machine learning tool for microscopy pixel classification. Bioinformatics. 2017;33(15):2424-2426.

21. Berg S, Kutra D, Kroeger T, Straehle CN, Kausler BX, Haubold C, et al. ilastik: interactive machine learning for (bio)image analysis. Nature Methods. 2019;

22. Cockburn K, Rossant J. Making the blastocyst: lessons from the mouse. The Journal of Clinical Investigation. 2010;120(4):995-1003.

23. Plusa B, Frankenberg S, Chalmers A, Hadjantonakis AK, Moore CA, Papalopulu N, et al. Downregulation of Par3 and aPKC function directs cells towards the ICM in the preimplantation mouse embryo. Journal of Cell Science. 2005;118(3):505-515.

24. Hirate Y, Hirahara S, Inoue KI, Suzuki A, Alarcon VB, Akimoto K, et al. Polarity-dependent distribution of angiomotin localizes Hippo signaling 
in preimplantation embryos. Current biology.

2013;23(23791731):1181-1194.

25. Ralston A, Cox BJ, Nishioka N, Sasaki H, Chea E, Rugg-Gunn P, et al. Gata3 regulates trophoblast development downstream of Tead4 and in parallel to Cdx2. Development. 2010;137(3):395-403.

26. Cockburn K, Biechele S, Garner J, Rossant J. The Hippo Pathway Member Nf2 Is Required for Inner Cell Mass Specification. Current Biology. 2013;23(13):1195 - 1201.

27. Frum T, Murphy TM, Ralston A. HIPPO signaling resolves embryonic cell fate conflicts during establishment of pluripotency in vivo. eLife. 2018;7(30526858):e42298.

28. Nishioka N, ichi Inoue K, Adachi K, Kiyonari H, Ota M, Ralston A, et al. The Hippo Signaling Pathway Components Lats and Yap Pattern Tead4 Activity to Distinguish Mouse Trophectoderm from Inner Cell Mass. Developmental Cell. 2009;16(3):398 - 410.

29. Kjær S, Linch M, Purkiss A, Kostelecky B, Knowles P, Rosse C, et al. Adenosine-binding motif mimicry and cellular effects of a thieno[2,3-d]pyrimidine-based chemical inhibitor of atypical protein kinase C isoenzymes. Biochemical Journal. 2013;451(2):329-342.

30. Rodriguez J, Peglion F, Martin J, Hubatsch L, Reich J, Hirani N, et al. aPKC Cycles between Functionally Distinct PAR Protein Assemblies to Drive Cell Polarity. Developmental Cell. 2020;42(4):400-415.e9. 
31. Aguilar-Aragon M, Elbediwy A, Foglizzo V, Fletcher GC, Li VSW, Thompson BJ. Pak1 Kinase Maintains Apical Membrane Identity in Epithelia. Cell Reports. 2020;22(7):1639-1646.

32. Yamada KM, Sixt M. Mechanisms of 3D cell migration. Nature Reviews Molecular Cell Biology. 2019;

33. Masuzzo P, Troys MV, Ampe C, Martens L. Taking Aim at Moving Targets in Computational Cell Migration. Trends in Cell Biology. $2016 ; 26(2): 88-110$.

34. Wolff C, Tinevez JY, Pietzsch T, Stamataki E, Harich B, Guignard L, et al. Multi-view light-sheet imaging and tracking with the MaMuT software reveals the cell lineage of a direct developing arthropod limb. eLife. 2018;7:e34410.

35. Pietzsch T, Preibisch S, Tomančák P, Saalfeld S. ImgLib2-generic image processing in Java. Bioinformatics. 2012;28(22):3009-3011.

36. Haase R, Royer LA, Steinbach P, Schmidt D, Dibrov A, Schmidt U, et al. CLIJ: GPU-accelerated image processing for everyone. Nature Methods. 2020;17(1):5-6. 
bioRxiv preprint doi: https://doi.org/10.1101/2020.10.15.340810; this version posted October 16, 2020. The copyright holder for this preprint (which was not certified by peer review) is the author/funder. All rights reserved. No reuse allowed without permission.

A

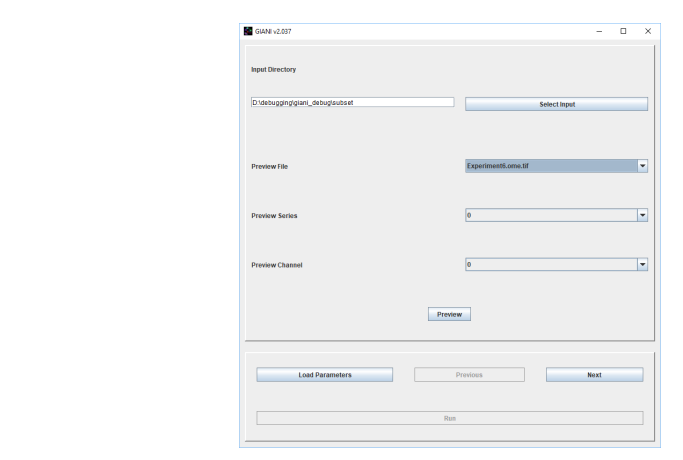

C

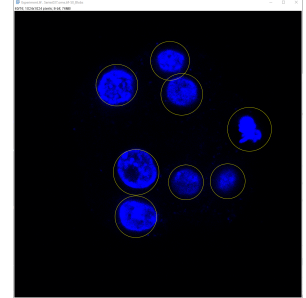

D

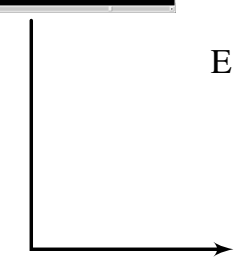

I

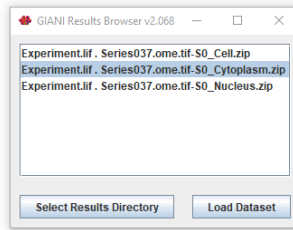

E

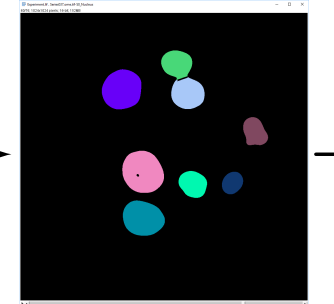

$\downarrow$

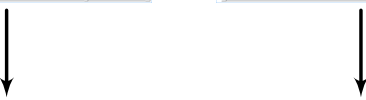

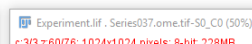

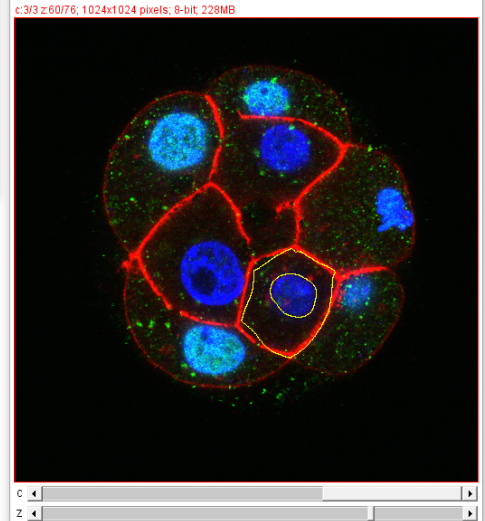

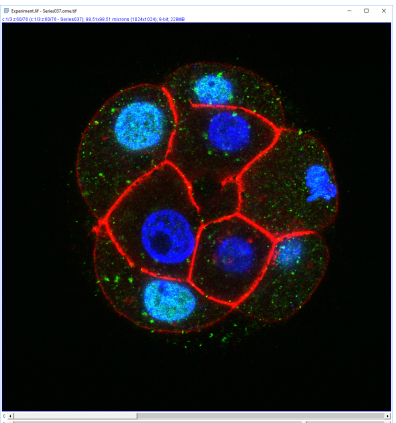

$\mathrm{F}$

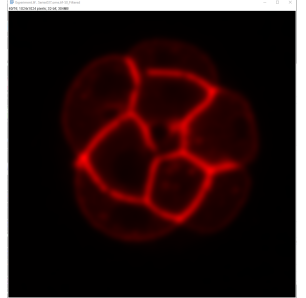

G

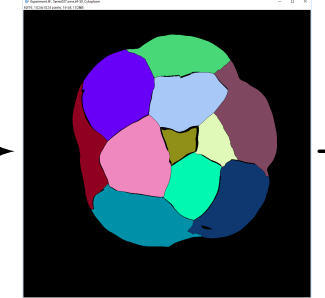

$\mathrm{H}$
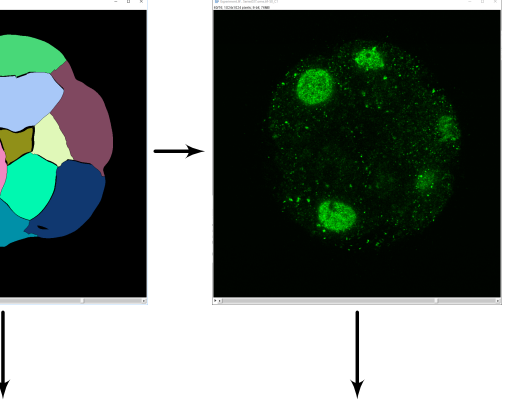

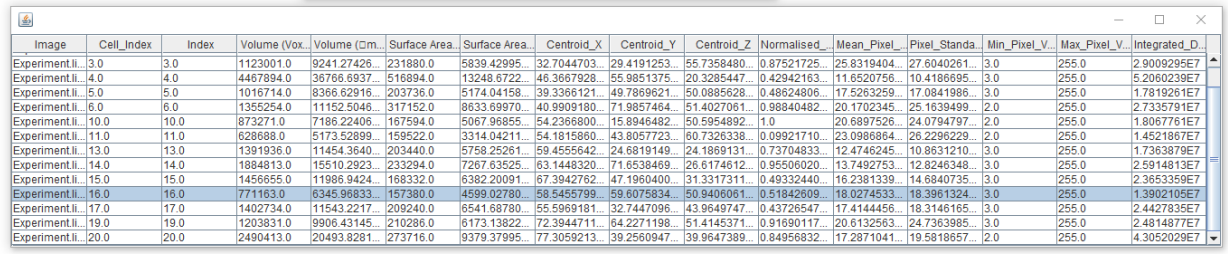

Figure 1 
bioRxiv preprint doi: https://doi.org/10.1101/2020.10.15.340810; this version posted October 16, 2020. The copyright holder for this preprint (which was not certified by peer review) is the author/funder. All rights reserved. No reuse allowed without permission.

A

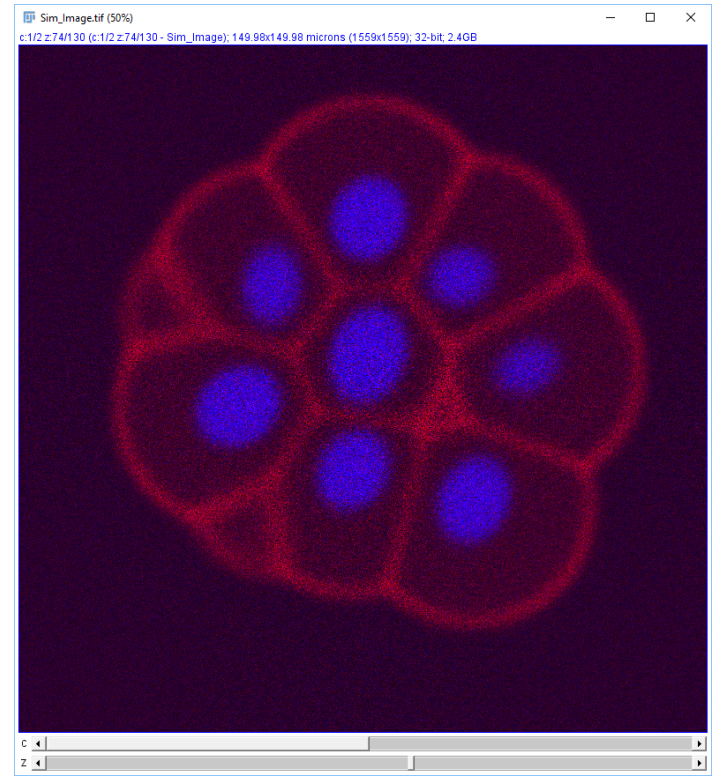

C

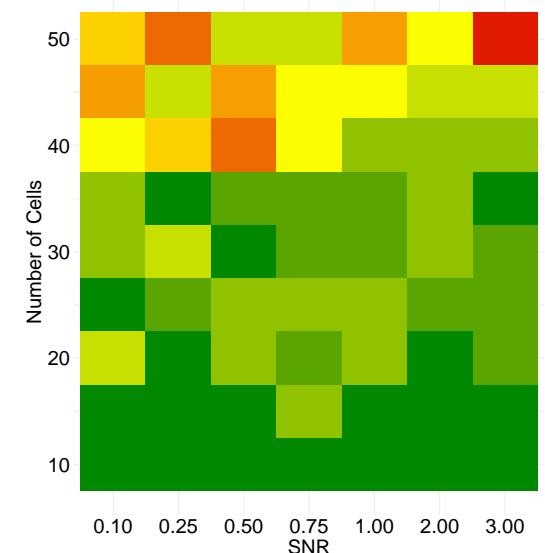

F

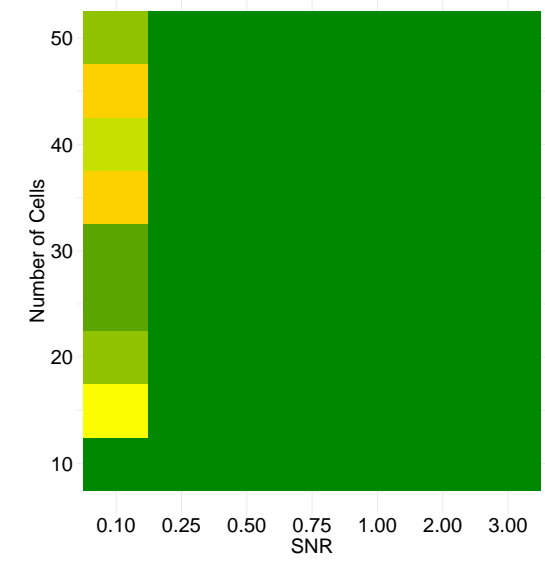

D

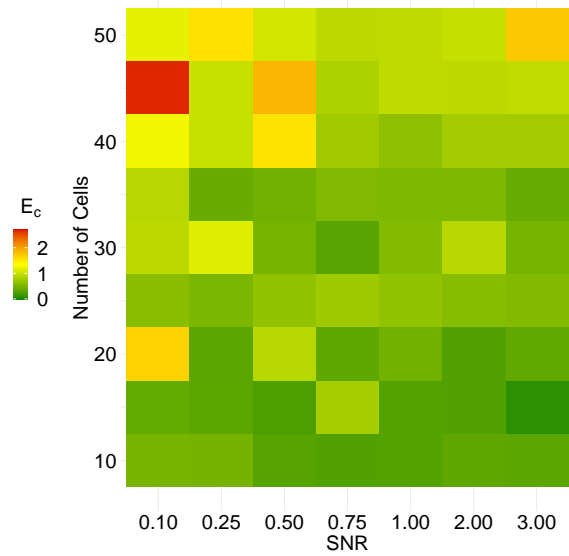

G

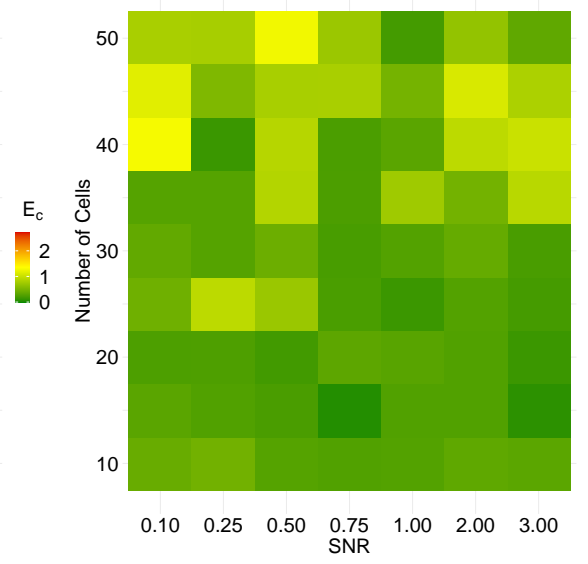

E

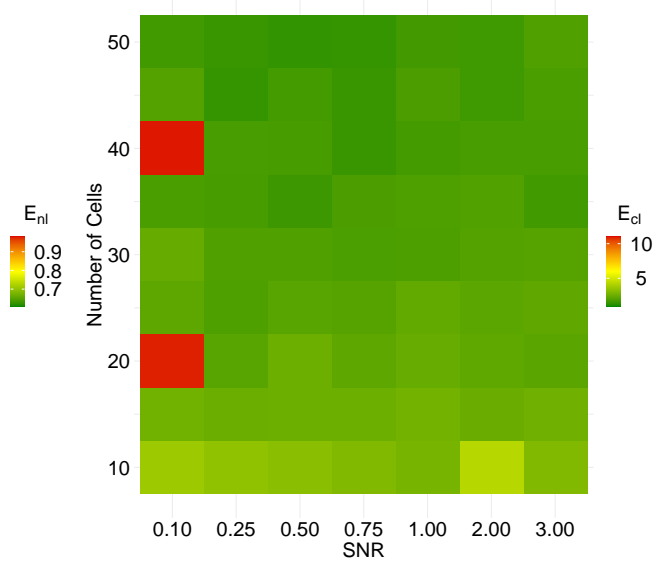

$\mathrm{H}$

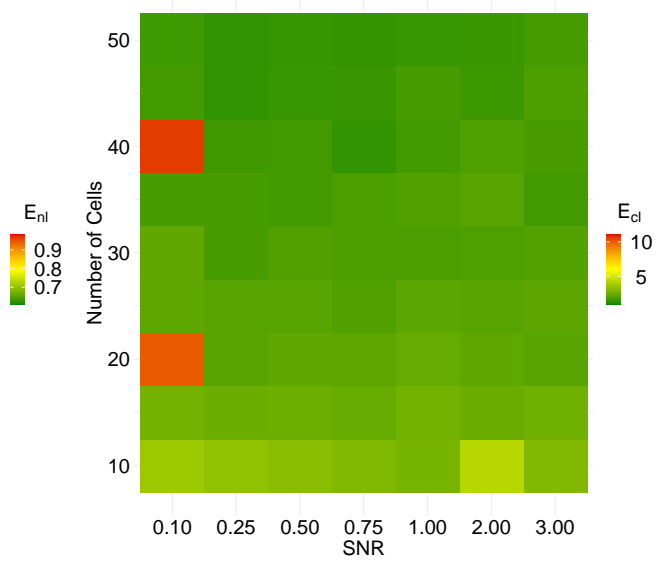

Figure 2 
bioRxiv preprint doi: https://doi.org/10.1101/2020.10.15.340810; this version posted October 16, 2020. The copyright holder for this preprint (which was not certified by peer review) is the author/funder. All rights reserved. No reuse allowed without permission.

A

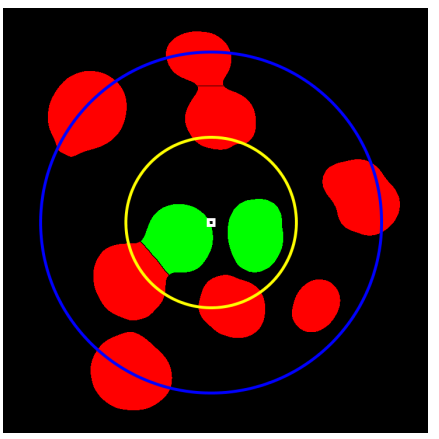

D

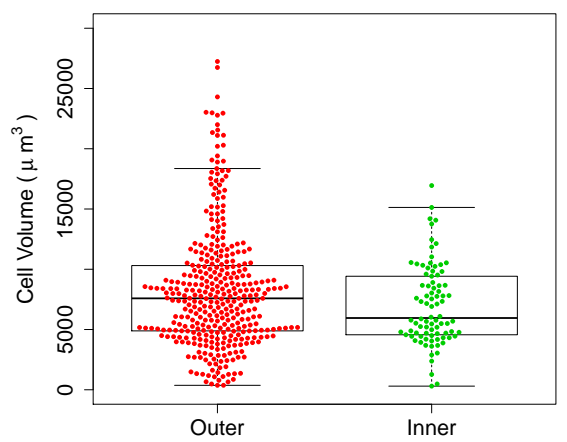

G

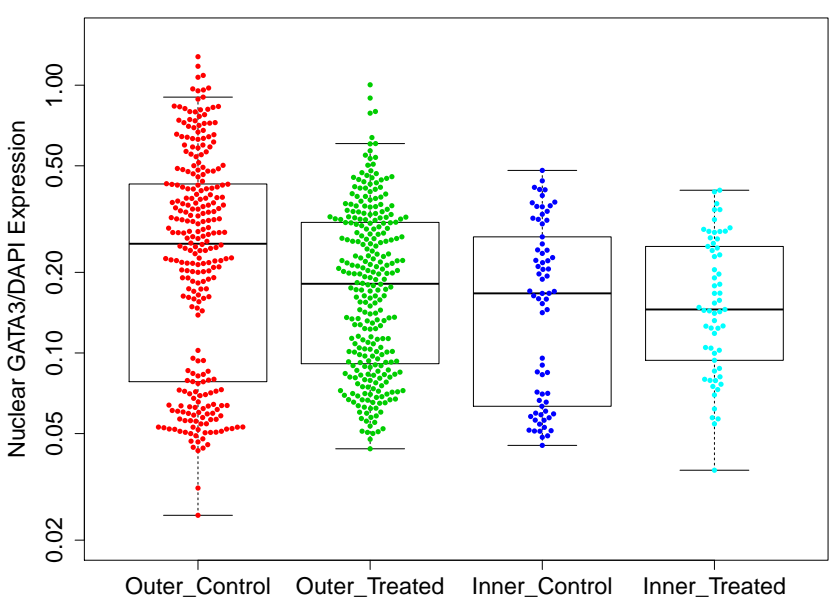

C
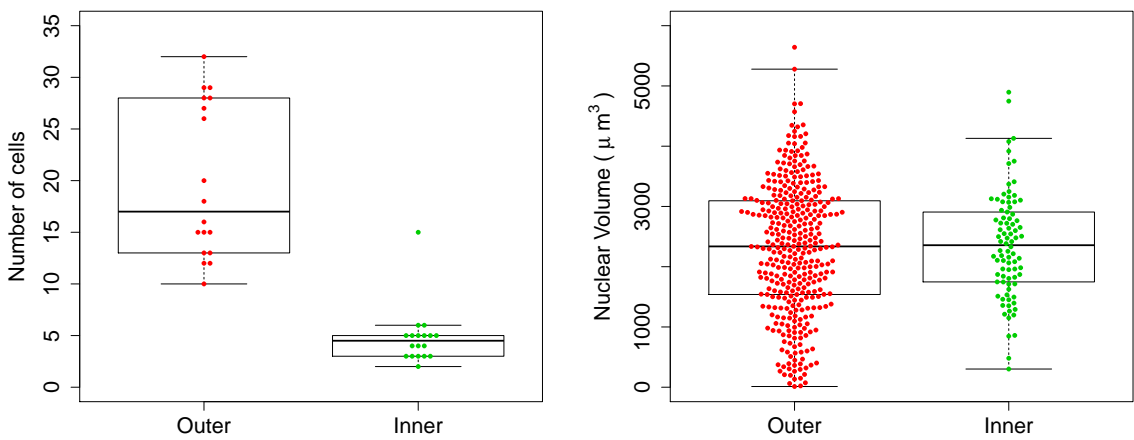

F
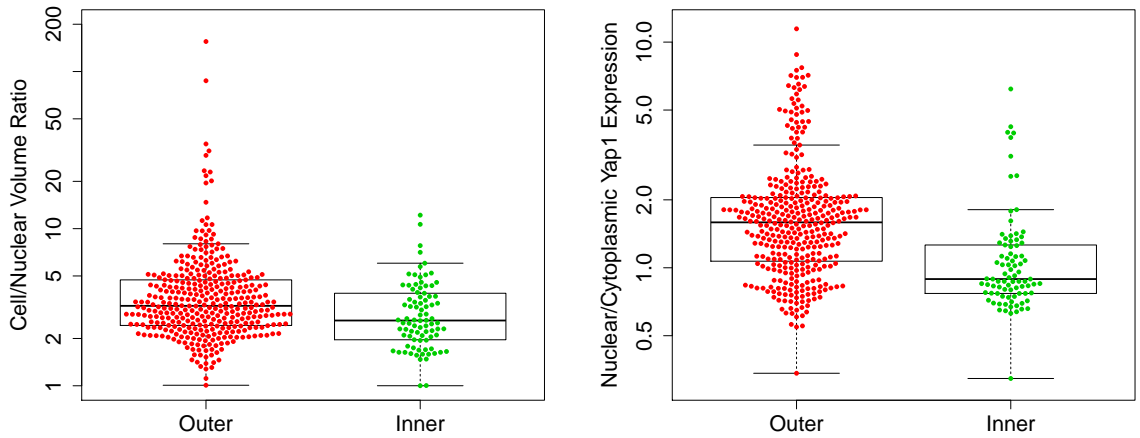

H

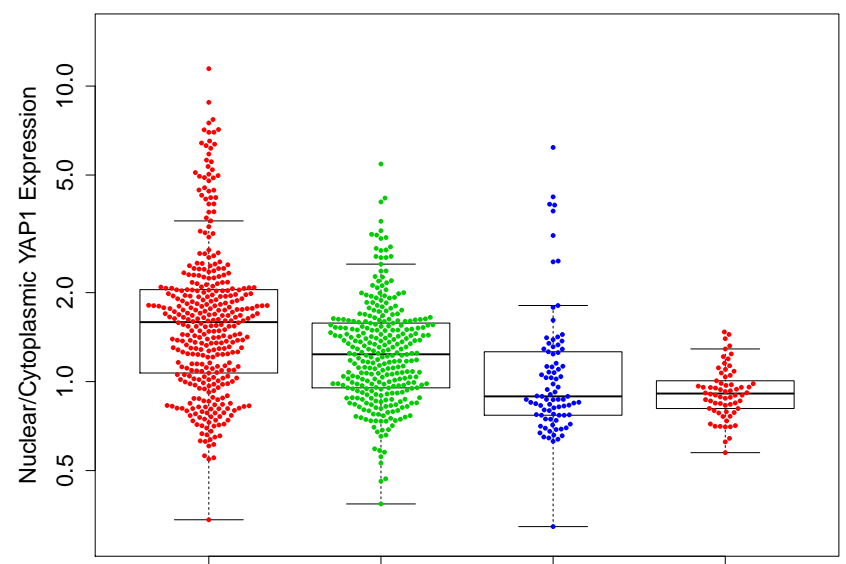

Outer_Control Outer_Treated Inner_Control Inner_Treated 